\title{
Visualization of Impurities on the Surface of Frozen Samples by Environmental Scanning Electron Microscopy
}

\author{
${\text { L’ubica Vetráková }{ }^{1} \text {, Vilém Neděla }{ }^{1} \text { and Jiří Runštuk }}^{1}$ \\ 1. Environmental Electron Microscopy Group, Institute of Scientific Instruments of the Czech Academy \\ of Science, Brno, Czech Republic.
}

Most of the chemical compounds soluble in water are very poorly soluble in ice. Therefore, when solution is freezing, impurities (solutes) are expelled from the growing ice lattice into unfrozen portion of the solution; they are freeze-concentrated in veins between ice crystals [1], (micro)pockets within the ice structure [2], or puddles and grain boundary grooves on the ice surface [3]. The location of the impurities within a frozen sample plays an important role in their reactivity and their availability, as for the impurities located close to the ice surface gas-phase oxidants are more easily accessible, and they can be released back to the environment more readily in comparison with the impurities buried in the frozen bulk [2].

In this study we utilized environmental scanning electron microscope (ESEM) to visualize the impurities located on the surface of frozen samples; the effect of freezing technique on the location and abundance of impurities on the surface of frozen samples was examined. $\mathrm{CsCl}$ was chosen as a model impurity due to its resemblance to $\mathrm{NaCl}$ that is the far most abundant salt present in seawater, and very good contrast between $\mathrm{CsCl}$ brine and ice when the detector of backscattered electrons (BSE), sensitive to atoms with large atomic number, was used. The frozen samples were prepared from $0.05 \mathrm{M} \mathrm{CsCl}$ solution by following freezing methods: (A) freezing a sample by gradual decrease of temperature (nucleation temperature was about $-18{ }^{\circ} \mathrm{C}$ ); (B) freezing a sample at $-2{ }^{\circ} \mathrm{C}$ when seeding was applied; (C) spraying very fine droplets of the solution into a vessel containing liquid nitrogen (LN); (D) freezing a sample in LN and its fragmentation to reveal interior of the sample. The samples were imaged in the ESEM AQUASEM II at the temperature of $-25^{\circ} \mathrm{C}$, pressure of $500-700 \mathrm{~Pa}$ and the beam energy of $20 \mathrm{keV}$.

The representative images of the variously prepared frozen samples are depicted in Figure 1 . There was a distinct difference in the size of ice crystals when various freezing methods were used; ice crystals in the sample B were much larger in comparison with the crystals in the samples A and C. The size of crystals is dependent on the freezing rate (rate of post-nucleation ice crystal growth), with faster freezing rates promoting a high number of small ice crystals and slower freezing rates promoting few large ice crystals [4]. Apparently, the supercooling and the freezing in LN promoted high freezing rates, while seeding at the temperature just below the freezing point resulted in much lower freezing rate. Although the overall concentration of $\mathrm{CsCl}$ in the samples was constant, the amount of $\mathrm{CsCl}$ on the surface of the frozen samples and its partition between grain boundary grooves and brine puddles differed depending on the freezing method. In the fast frozen samples ( $\mathrm{A}$ and $\mathrm{C}$ ) $\mathrm{CsCl}$ brine was mainly located in the grain boundary grooves, while it formed puddles on the surface of slowly frozen sample B. We suppose, as slow freezing allows more thorough freeze-concentration of impurities, these are expelled from the growing ice to its outer surface (the ice-air interface). On the other hand, fast freezing aborts the freezeconcentration process, therefore the impurities are expelled just to the boundaries of individual ice grains. One would expect, however, that the surface of the slowly frozen sample B would be covered with brine in much larger extent in comparison with the fast frozen samples due to more extensive freeze-concentration; this was not the case. We suppose, as the sample B was seeded from the top, most 
of the salt was expelled from the growing ice towards the bottom and the edges of the sample and only a small part of the salt remained on the top surface. Apparently, location of impurities in the frozen sample is not only dependent on the freezing rate but also on the directionality of freezing. Analogously, by immersing a sample into LN the freezing was directed from the sample surface inwards. Therefore, expelling of $\mathrm{CsCl}$ from the advancing freezing front resulted in $\mathrm{CsCl}$ trapped in the interior of the frozen sample (D).

In this study, ESEM was demonstrated to be a useful tool for visualization of impurities on the surface of frozen samples due to the high contrast between ice and $\mathrm{CsCl}$ brine. The presented results suggest that the freezing rate and directionality are important factors for the location of impurities within the frozen samples. All requests for poster presentations will be honoured [5].

\section{References:}

[1] JR Blackford et al, Proc. Inst. Mech. Eng. Part L-Journal Mater. Appl. 221 (2007), p. 151.

[2] T Hullar et al, C. Cryosphere 10 (2016), p. 2057.

[3] J Krausko et al, J. Langmuir 30 (2014), p. 5441.

[4] JC Kasper et al, Eur. J. Pharm. Biopharm. 78 (2011), p. 248.

[5] The research was supported by the MEYS CR (LO1212), its infrastructure by the MEYS CR and the EC (CZ.1.05/2.1.00/01.0017) and by the CAS (RVO: 68081731).

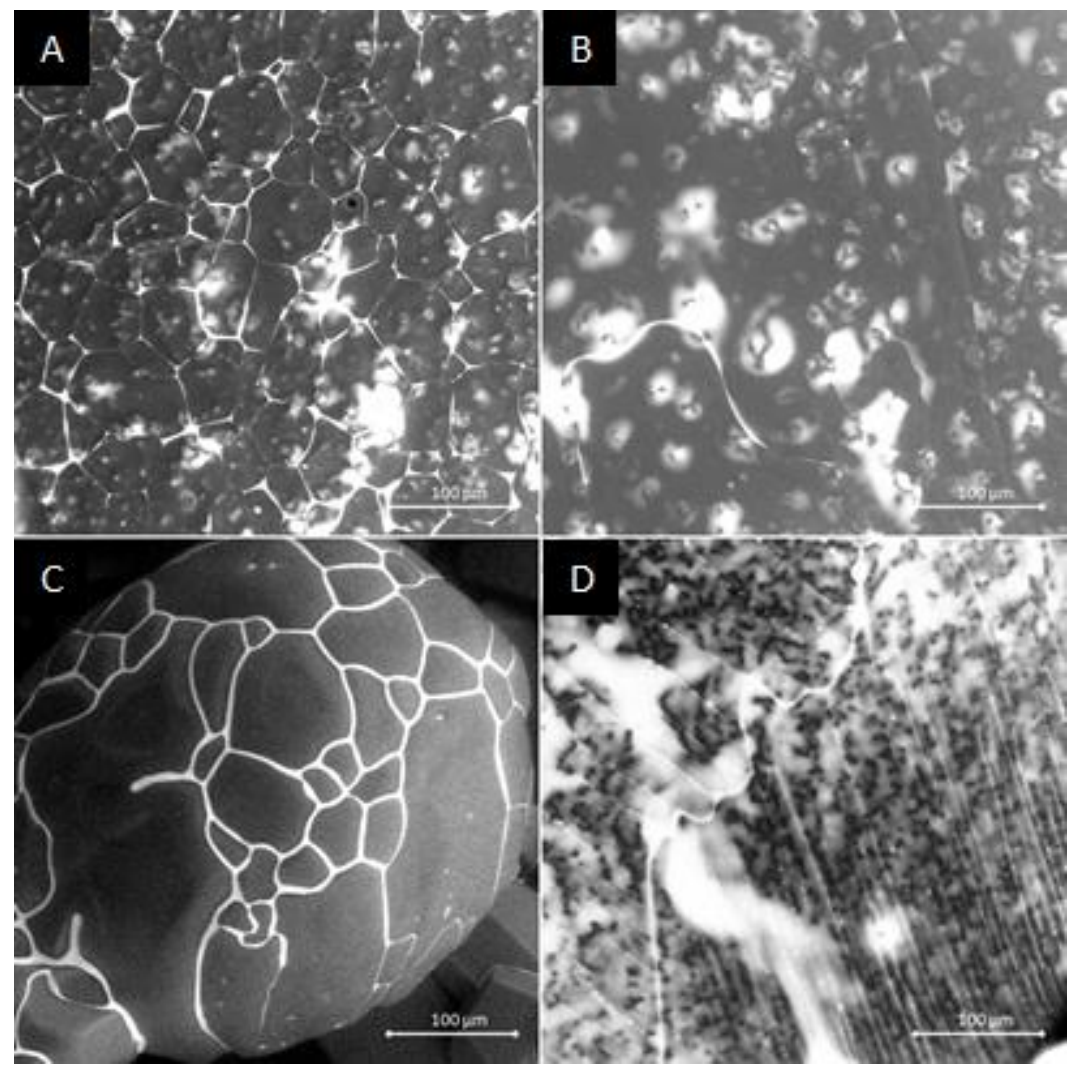

Figure 1. ESEM images of frozen samples prepared from $0.05 \mathrm{M} \mathrm{CsCl}$ solution by spontaneous freezing of a sample supercooled to $-18^{\circ} \mathrm{C}(\mathrm{A})$, freezing of a sample at $-2{ }^{\circ} \mathrm{C}$ with applied seeding (B), spraying fine droplets into $\mathrm{LN}(\mathrm{C})$, and the interior of a sample frozen in LN revealed after the sample fragmentation (D). Grey areas represent ice, white colour represents $\mathrm{CsCl}$ brine. 